This paper has been published under reference:

Zivanovic, S., Russell, J. and Racic, V. (2019) Vibration Performance of a Lightweight FRP Footbridge under Human Dynamic Excitation. IMAC-XXXVII, Orlando, Florida, 28 - 31 January.

\title{
Vibration Performance of a Lightweight FRP Footbridge under Human Dynamic
} Excitation

\author{
Stana Živanović ${ }^{1}$, Justin M. Russell ${ }^{2}$, Vitomir Racic ${ }^{3}$ \\ ${ }^{1}$ College of Engineering, Mathematics and Physical Sciences, University of Exeter, UK \\ ${ }^{2}$ School of Engineering, University of Warwick, UK \\ ${ }^{3}$ Department of Civil and Environmental Engineering, Politecnico di Milano, Italy
}

\begin{abstract}
Fibre-reinforced polymer (FRP) composites are increasingly used as main load bearing materials in design of pedestrian bridges. The FRP footbridges are typically characterised by high strength, and relatively low mass and stiffness. These properties could lead to excessive vibration response under human-induced dynamic loading. This paper studies dynamic performance of a $19.8 \mathrm{~m}$ long, simply supported, FRP footbridge exposed to walking and jogging. Moreover, the vibration response of this bridge is compared and critically evaluated against the response of an equivalent, in terms of natural frequency and span length, composite steel-concrete structure. The main factors that drive the vibration performance of the FRP structure are discussed and some recommendations for vibration serviceability checks are made.
\end{abstract}

Keywords: FRP composites, Footbridge, Walking, Jogging, Vibration

\section{INTRODUCTION}

Fiber-reinforced polymer (FRP) structures are known to be responsive to dynamic excitation due to their relatively low weight and stiffness. This paper investigates responsiveness of an FRP shallow-truss footbridge structure having $19.8 \mathrm{~m} x$ $2.1 \mathrm{~m}$ deck area. The bridge spans $16.8 \mathrm{~m}$, and it has the first vertical flexural vibration mode at $2.53 \mathrm{~Hz}$. The modal mass for this mode is $650 \mathrm{~kg}$ and damping ratio is $1.69 \%$ [1]. The performance of this bridge, referred to FRPB hereafter, will be compared against a classical composite steel-concrete bridge, referred to as CSCB, of similar size and natural frequency. The CSCB has a deck area of $19.9 \mathrm{~m}$ x $2.0 \mathrm{~m}$. It spans $16.2 \mathrm{~m}$. The fundamental vertical flexural vibration mode has natural frequency of $2.44 \mathrm{~Hz}$, modal mass of $7700 \mathrm{~kg}$ and damping ratio around $0.45 \%$ [2]. Both bridges are slender simply supported structures with overhangs on both sides. The mode shape for the vertical flexural mode that is studied in this paper is a typical wave with movement between the supporting points being $180^{\circ}$ out-of phase with the movement of overhangs $[1,2]$. The slenderness (span length to truss height or cross section depth) ratio is 35 for FRPB and 46 for CSCB. The two bridges are shown in Figure 1. Next two sections explain and compare performance of the two bridges under pedestrian and jogger excitation, respectively. This is followed by summary of main conclusions from the study.

\section{WALKING}

Vibration response to dynamic excitation by a person walking was calculated using a stochastic model of the dynamic force developed by Racic and Brownjohn [3]. The excitation by one person at a time only was considered in this paper. Pacing frequencies starting from extremely low $1.2 \mathrm{~Hz}$ to extremely high $2.6 \mathrm{~Hz}$ were considered (in frequency steps of $0.1 \mathrm{~Hz}$ ). For each frequency 100 forces with different waveforms were generated to represent intra-subject variations. The mean and standard deviation for peak vibration response at the mid-span for each pacing frequency was found. The weight of the pedestrians is assumed to be $700 \mathrm{~N}$; this parameter has not been treated as random in order to isolate the influence of the most significant locomotion parameter - pacing rate - on the vibration response. The influence of the pedestrian weight is simple to gauge later on, if necessary, due to the response being directly proportional to the pedestrian weight.

The left part of Figure 2 shows the mean values of the peak response as a function of pacing frequency for the two bridges, as well as mean \pm STD, where STD represents the standard deviation of the peak response. Both bridges are most vulnerable to pacing rates between $2.4 \mathrm{~Hz}$ and $2.6 \mathrm{~Hz}$ since the forces in this range are either close to the natural frequency or matching it. 
This paper has been published under reference:

Zivanovic, S., Russell, J. and Racic, V. (2019) Vibration Performance of a Lightweight FRP Footbridge under Human Dynamic Excitation. IMAC-XXXVII, Orlando, Florida, 28 - 31 January.

The FRPB is exceptionally responsive, with the strongest mean peak acceleration of $10 \mathrm{~m} / \mathrm{s}^{2}$ overcoming the acceleration of gravity. The strongest mean response of CSCB is comparatively much lower at $1.2 \mathrm{~m} / \mathrm{s}^{2}$. The main cause of the difference in the response is that the modal mass of the FRPB is one order of magnitude lower than for CSCB. The differences in damping ratio and natural frequencies further combine to influence the response, but this influence is relatively small compared with the influence of the modal mass.
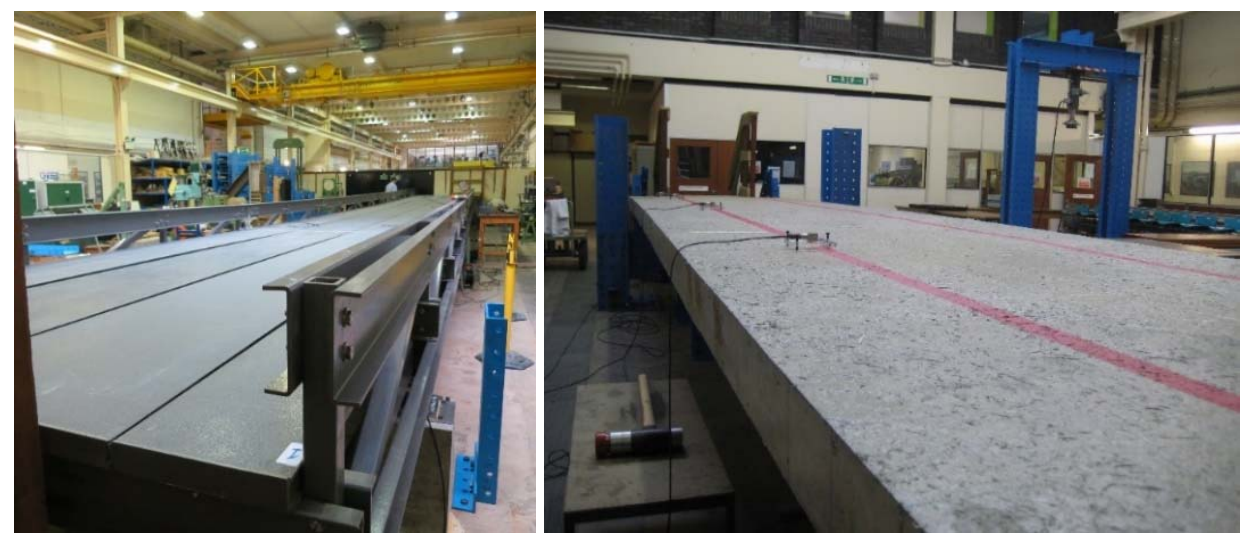

Figure 1: Photographs of the two footbridges. Left. FRPB. Right. CSCB.
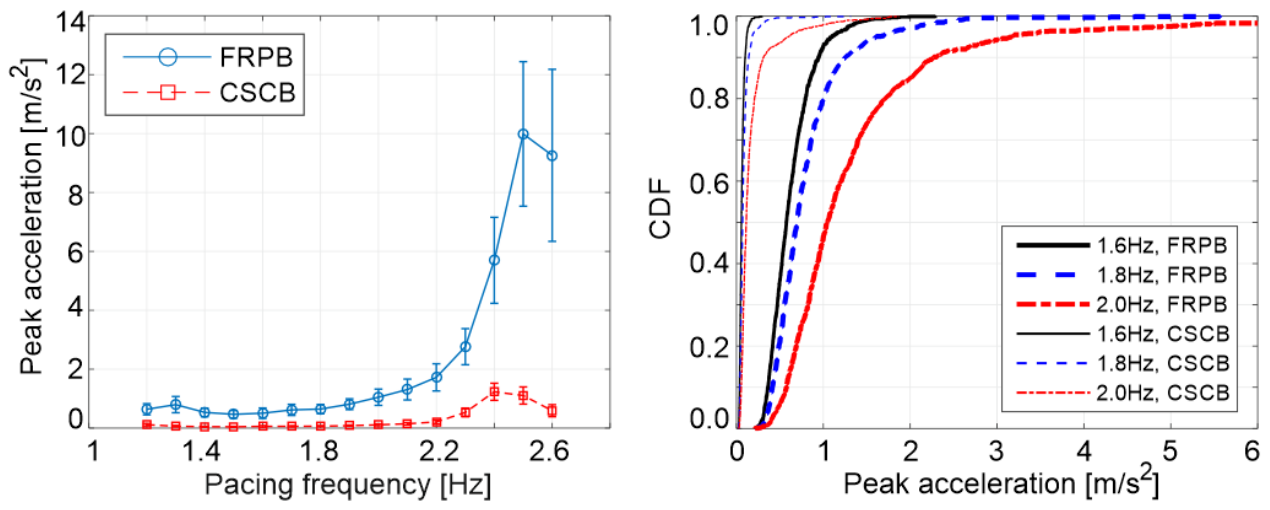

Figure 2: Walking excitation: Left: Peak acceleration (mean \pm STD) vs pacing frequency. Right: CDF for peak acceleration responses.

Whilst the walking at a frequency close to the natural frequency results in the strongest response of the two bridges, these frequencies are not frequently occurring in human population. To account for the probability of walking at any particular frequency, the vibration response was simulated for 1000 individuals. Pacing frequency for each person was drawn from a normal distribution $\mathcal{N}(1.6 \mathrm{~Hz}, 0.2 \mathrm{~Hz})$, where $1.6 \mathrm{~Hz}$ and $0.2 \mathrm{~Hz}$ represent the mean value and the standard deviation, respectively. This particular distribution represents a relatively slow pedestrian population, which might be encountered on a bridge in, say, a leisure park. In addition, populations with two additional pacing distributions: $\mathcal{N N}^{\circ}(1.8 \mathrm{~Hz}, 0.2 \mathrm{~Hz})$ and $\mathcal{N}^{\circ}$ $(2.0 \mathrm{~Hz}, 0.2 \mathrm{~Hz})$, where the latter represent a population in hurry, are considered. A cumulative distribution function (CDF) for the peak responses on both bridges are shown in the right part of Figure 2. As expected, when the mean pacing frequency is closer to the structural natural frequency, the response is larger. However, the probability of exciting the resonance on FRPB even in the case of the fastest pedestrian population is still not very large, and despite maximum response exceeding $10 \mathrm{~m} / \mathrm{s}^{2}$ (these values are not presented in the figure), the probability of exceeding, say, $2.5 \mathrm{~m} / \mathrm{s}^{2}$ is less than $10 \%$.

\section{JOGGING}

Similar information to that in Figure 2 is shown in Figure 3 in relation to single jogger's excitation. The jogging force is generated using the model developed in [4]. The jog frequency is varied from $2.5 \mathrm{~Hz}$ to $3.4 \mathrm{~Hz}$, and 100 individuals are simulated for each case. The response of FRPB is again one order of magnitude larger than that of CSCB. The response even 
This paper has been published under reference:

Zivanovic, S., Russell, J. and Racic, V. (2019) Vibration Performance of a Lightweight FRP Footbridge under Human Dynamic Excitation. IMAC-XXXVII, Orlando, Florida, 28 - 31 January.

to non-resonance frequencies is high and at least $4 \mathrm{~m} / \mathrm{s}^{2}$ due to higher energy of the jogging action compared with walking. Considering population of 1000 joggers (traversing the bridge one at a time) with the jog frequency drawn from $\mathcal{N}^{\circ}(2.4 \mathrm{~Hz}$, $0.25 \mathrm{~Hz})$ and $\mathcal{N}(2.8 \mathrm{~Hz}, 0.25 \mathrm{~Hz})$, the CDFs (on the right-hand side of the figure) illustrate that the large majority of joggers would cause vibrations larger than even the most relaxing acceleration limit of $2.5 \mathrm{~m} / \mathrm{s}^{2}$
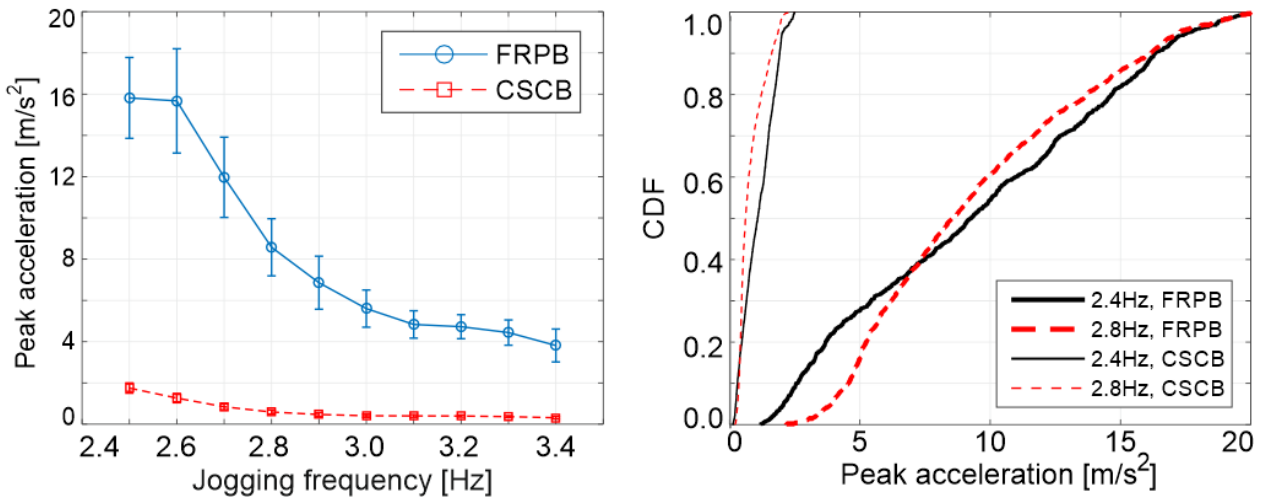

Figure 3: Jogging excitation: Left: Peak acceleration (mean \pm STD) vs jogging frequency. Right: CDF for peak acceleration responses.

\section{CONCLUSIONS}

The paper compares responses of an FRP and a non-FRP bridge to pedestrians and joggers. The response in the first vertical flexural mode is shown only. It demonstrates that for all loading scenarios considered, FRPB is one order of magnitude more responsive than $\mathrm{CSCB}$, primarily due to having modal mass that is almost 12 times lower. Having damping ratio that is 3.8 time larger is a positive feature, but it, in comparison, has less influence on the vibration response. The results show the importance of considering probability of occurrence for pacing rates. In addition, the FRP bridges are more vulnerable to jogging excitation even when the resonance does not occur. This type of analysis should, in general, be conducted for all vibration modes that are of interest. Responsiveness of the modes above $5 \mathrm{~Hz}$ should also be checked, since they are likely to be excited by weaker (than first) harmonics of the excitation force due to low modal masses of FRP structures. Note that both bridges studied here have been designed to be lively for research purposes, and the actual structures of similar span are almost certain to vibrate less. Still comparison of the relative behavior is revealing. Given the high vibration levels generated on the FRPB, it is almost certain that both pedestrians and joggers would interact with the structure in some way. The actual vibration of this bridge, therefore, might be different from the predications presented in this study. The next step in this research is to quantify if and how the experimental responses differ from those predicted, and develop a model to describe the interaction phenomenon.

\section{ACKNOWLEDGEMENTS}

This research work was supported by the UK Engineering and Physical Sciences Research Council [grant number EP/M021505/1: Characterising dynamic performance of fibre reinforced polymer structures for resilience and sustainability].

\section{REFERENCES}

[1] Russell, J.M., Mottram, J.T., Živanović, S. and Wei, X. (2019) Design and performance of a bespoke lively all-FRP footbridge. Proceedings of IMAC-XXXVII, Orlando, Florida, 28 -31 January.

[2] Dang, H. V. and Živanović, S. (2016) Influence of low-frequency vertical vibration on walking locomotion. Journal of Structural Engineering, 142 (12).

[3] Racic, V. and Brownjohn, J.M.W. (2011) Stochastic model of near-periodic vertical loads due to humans walking. Advanced Engineering Informatics, 25 (2).

[4] Racic, V. and Morin, J.B. (2014) Data-driven modelling of vertical dynamic excitation of bridges induced by people running. Mechanical Systems and Signal Processing. 43. 